\title{
Evidence of the Miniband Formation in InGaAs/InP Superlattices
}

\author{
Yu. A. Pusep ${ }^{1}$, A. de Giovanni Rodrigues ${ }^{2}$, J. C. Galzerani ${ }^{2}$, \\ ${ }_{1}^{1}$ Instituto de Fisica de São Carlos, Universidade de São Paulo, 13560-970 São Carlos, SP, Brazil. \\ ${ }^{2}$ Departamento de Fisica, Universidade Federal de São Carlos, CP 676,13565-905, São Carlos, SP, Brazil \\ D. Comedi, and R. R. LaPierre \\ Centre for Emerging Device Technologies, McMaster University, Hamilton, Ontario, L8S 4L7, Canada
}

Received on 8 December, 2005

\begin{abstract}
The formation of the miniband electron energy structure was explored in doped InGaAs/InP superlattices with different periods. The analysis of the Raman data allowed us to conclude that in spite of the defect structure of the layers constituting the superlattices, their super-periodicity was well defined. The quantitative proof of the conditions for break-down of the Raman selection rules is presented: the emergence of the selection rules of the coupled plasmon- $L O$ phonon vibrations was demonstrated to occur due to the increase of their coherence lengths. In addition, the expected anisotropy of the effective electron masses was found by high-field magnetoresistance.
\end{abstract}

Keywords: Superlattices; Plasmons; Raman scattering

InGaAs/InP heterostructures present an important class of semiconductor materials with possible applications in infrared optoelectronics. Moreover, a large $g$-factor found in these materials [1] makes them promising in the field of spintronics and quantum information processing. A manipulation of the electronic properties (including $g$-factor engineering) important in device applications is possible in superlattices (SLs) structures consisting of periodic sequences of the layers made of two different semiconductor materials. In such structures the dynamic electron properties can be tuned in the direction perpendicular to the layers by a variation of their thicknesses or compositions. However, to the best of our knowledge until now an evidence of the formation of the superlattice bandstructure in the InGaAs/InP SLs was only recently obtained by high-field magnetotransport in Ref.[2]. The photoluminescence data presented in this reference also indicated the existence of the minibands.

In this work we explored the Raman scattering of the coupled plasmon- $L O$ phonon modes propagating perpendicular to the layers of the short-period doped InGaAs/InP SLs. The manifestation of the coupled modes in the short-period SLs was assigned to the formation of the miniband energy structure caused by the SL periodicity. We also found an additional indication of the miniband formation by high-magnetic field magnetoresistance measurements. Moreover, a considerable effect of the super-periodicity on the Raman selection rules of the coupled modes was observed.

A total number of 30 periods of the $\left(I n_{0.53} G a_{0.47} A s\right)_{m}(\operatorname{InP})_{m}$ SLs with $m=6,8,10,15$, and 68 $M L$ (excepting the SL with $m=68$ which has 20 periods), where $n$ is the thickness of the layers expressed in monolayers $(1 M L=2.9 \stackrel{\circ}{A})$, were grown on semi-insulating (001) InP substrates by molecular beam epitaxy. The high-resolution $\mathrm{X}$-ray diffraction showed the variation in the period of our SLs of about $0.3 \%$. The barriers of the SLs were doped by $\mathrm{Si}$ in order to form a gas of degenerate electrons with the density determined by the Hall measurements $5.0 \times 10^{17} \mathrm{~cm}^{-3}$. The doping was kept the same for all the SLs reported here.
The magnetoresistance measurements were performed on samples patterned into an active area of $4 x 4 \mathrm{~mm}$. The Ohmic contacts were fabricated by depositing indium. The transport measurements parallel to the surface of the samples were carried out at $T=4.2 \mathrm{~K}$ in the Van der Pauw geometry using a standard low-frequency $(5 \mathrm{~Hz})$ lock-in technique in a liquid $\mathrm{He}$ cryostat with the magnetic fields directed perpendicular to the current. The Raman scattering was collected from the surface of the samples at $T=10 \mathrm{~K}$ in the back-scattering configuration with a "Instruments S.A. T64000" triple grating spectrometer supplied with a CCD detector cooled by liquid nitrogen; the $5145 \stackrel{\circ}{A}$ line of an $A r^{+}$laser was used for nonresonant excitation.

The Raman spectra of the optical vibrational modes propagating along the growth direction measured in $z\left(x^{\prime} y^{\prime}\right) \bar{z}$ crossand $z\left(x^{\prime} x^{\prime}\right) \bar{z}$ parallel-polarized configurations of the backscattering, where $x^{\prime}, y^{\prime}, z$ are the crystallographic directions [110], [110] and [001] respectively, are shown in Fig. 1. In such a case, according to the Raman selection rules [3], the longitudinal optical lattice vibrations $(L O)$ are active in the cross-polarized configuration, while no optical modes contribute to the parallel-polarized Raman scattering. In the longperiod InGaAs/InP SL with the thicknesses of both layers equal to $68 M L$ the following vibrational modes were observed: the allowed $L O$ and the forbidden transversal optical (TO) vibration modes of the InGaAs wells (the InAs-like modes with the indices 1 and the $G a A s$-like modes with the indices 2, respectively) and the InP barriers (the modes with the indices 3). Moreover, the mode at $274 \mathrm{~cm}^{-1}$ may be identified as the $G a A s$-like interface $\left(I F_{1}\right)$ disorder activated mode. No selection rules were found in this SL. The absence of the Raman selection rules and the evidence of the forbidden $T O$ and $I F$ modes are manifestation of the significant violation of the translational symmetry in the bulk materials constituting the SL. At the same time, the expected Raman selection rules emerge when decreasing the period of the SLs and they are clearly determined in the SLs with the shortest periods $(m=6$ $M L$ and $8 M L$ ) for the longitudinal modes denoted as $L_{1}, L_{2}$ 


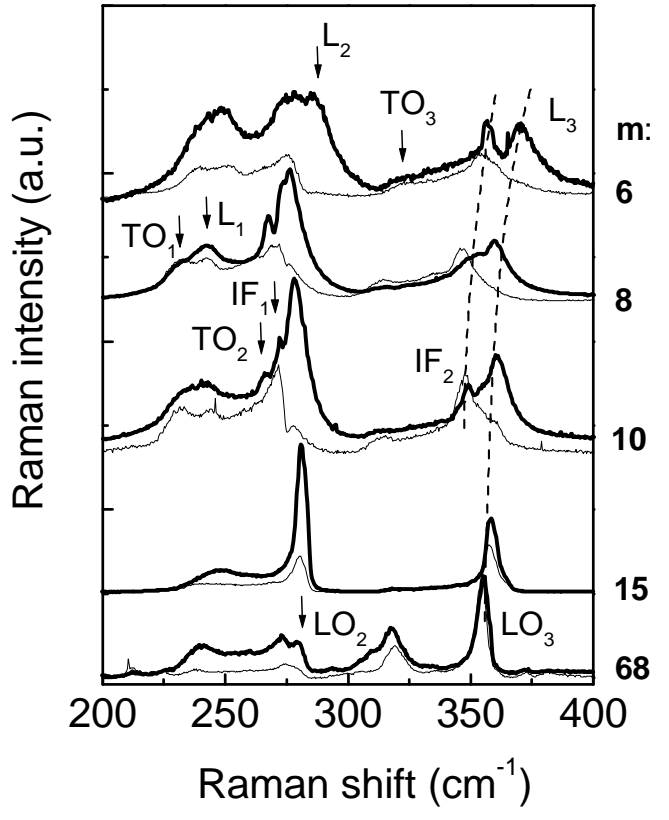

FIG. 1: Raman scattering measured at $T=10 K$ in the $(\operatorname{InGaAs})_{m}(\operatorname{In} P)_{m}$ superlattices with different periods. Thick and thin lines correspond to $z\left(x^{\prime} y^{\prime}\right) \bar{z}$ and $z\left(x^{\prime} x^{\prime}\right) \bar{z}$ scattering configurations respectively.

and $L_{3}$. Besides, considerable blue shifts of these longitudinal modes were found with decreasing SL period. The InPlike interface modes $\left(I F_{2}\right)$ detected in the short-period SLs at $350 \mathrm{~cm}^{-1}$ also reveal a similar blue shift, which indicates their mostly longitudinal character.

The observed effects can be caused by a formation of the miniband structure in the electron energy spectrum of the SLs due to the super-periodicity. The dynamic properties of the electrons propagating in the growth direction of a SL are determined by the miniband dispersion $E\left(k_{z}\right)$, where $k_{z}$ is the wave number along the growth $z$ direction. With decreasing period the miniband dispersion increases and as a consequence, the effective mass of the electrons propagating in the $z$ direction $\left(m_{z}\right)$ decreases resulting in the increase of the frequency of the plasmon oscillations polarized in the same direction according to $\omega_{p z}=\left(4 \pi e^{2} n / \varepsilon_{\infty} m_{z}\right)^{1 / 2}$, where $n$ is the electron concentration and $\varepsilon_{\infty}$ is the dielectric constant of the superlattice. The coupling between the plasmons and the optical phonons with the same polarization causes the blue shift of the longitudinal coupled plasmon- $L O$ phonon modes $L_{1}$, $L_{2}$ and $L_{3}$ observed in Fig. 1 and this shift increases with the increasing plasmon frequency. The variation of the effective mass is responsible for the observed blue shift when the electron concentration is fixed. In such a case, the blue shift of the coupled modes can serve as a manifestation of the formation of the miniband energy structure.

In order to prove the effect of the miniband dispersion on the shift of the coupled modes we calculated the miniband effective mass in all the SLs. The calculations were performed using the envelope function approximation [4] with the band

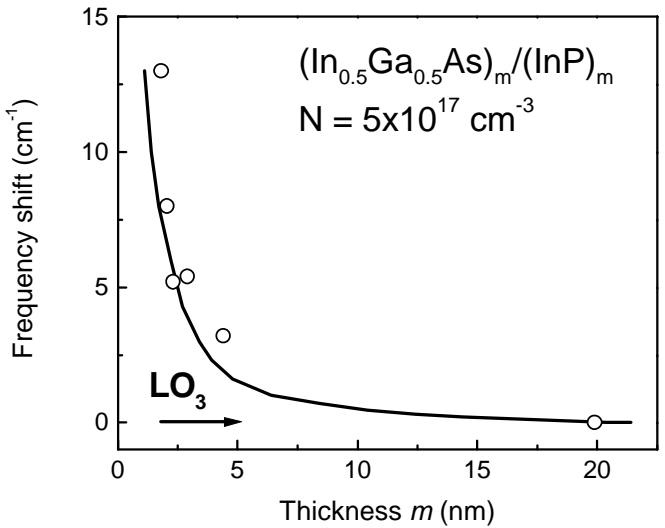

FIG. 2: Calculated (full line) and measured (open circles) frequency shifts of the $I n P$ coupled mode $\left(L_{3}\right)$ relative to the frequency of the InP phonon $\left(\mathrm{LO}_{3}\right)$ measured in the $(\operatorname{InGaAs})_{m}(\operatorname{InP})_{m}$ superlattice with different layer thicknesses $(m)$ and the same doping.

parameters taken from Ref.[5]. The frequencies of the coupled plasmon- $L O$ phonon modes were calculated as zeros of the corresponding diagonal component of the dielectric function tensor of a SL in the long wavelength approximation according to Ref.[6]. The calculated frequency shifts of the InPlike coupled mode $\left(L_{3}\right)$ together with the experimental data are shown in Fig. 2. A good agreement between the experimental and calculated results confirms that the blue shifts of the longitudinal modes were observed due to the miniband effect.

In addition, the miniband effect explains the emergence of the Raman selection rules observed for the coupled modes in the short-period SLs. As seen in Fig. 1, no selection rules expected for the longitudinal lattice modes were found in the long-period SLs due to the defect structure of the constituent bulk materials. Whereas, according to our data the longitudinal lattice vibrations transform to the coupled plasmon $L O$ phonon vibrations in the short-period SLs. The coupled modes usually reveal the Raman selection rules of the $L O$ phonons [3] indeed observed in the short-period SLs studied here. Raman scattering of the lattice vibrations exhibits corresponding selection rules when their spatial coherence length is much higher than the size of the crystal unit cell. We suppose that the coherence lengths of the optical phonons relevant to the SLs reported here are short enough to cause the breakdown of the Raman selection rules. Our data show that in the short-period SLs the electrons propagate over several periods and their collective excitations (plasmons) being coupled to the $L O$ phonons result in the increase of the correlation length of the coupled vibrations. As a consequence of the enhancement of the correlation length the Raman selection rules may appear.

In order to confirm the effect of the increase of the coherence length of the coupled plasmon-like vibrations on their Raman selection rules we determined and compared the coherence lengths of the optical phonons and of the coupled plasmon- $L O$ phonon modes. As it was demonstrated in Ref.[7], in the presence of disorder a finite coherence length of the excitations involved in Raman scattering causes the asym- 


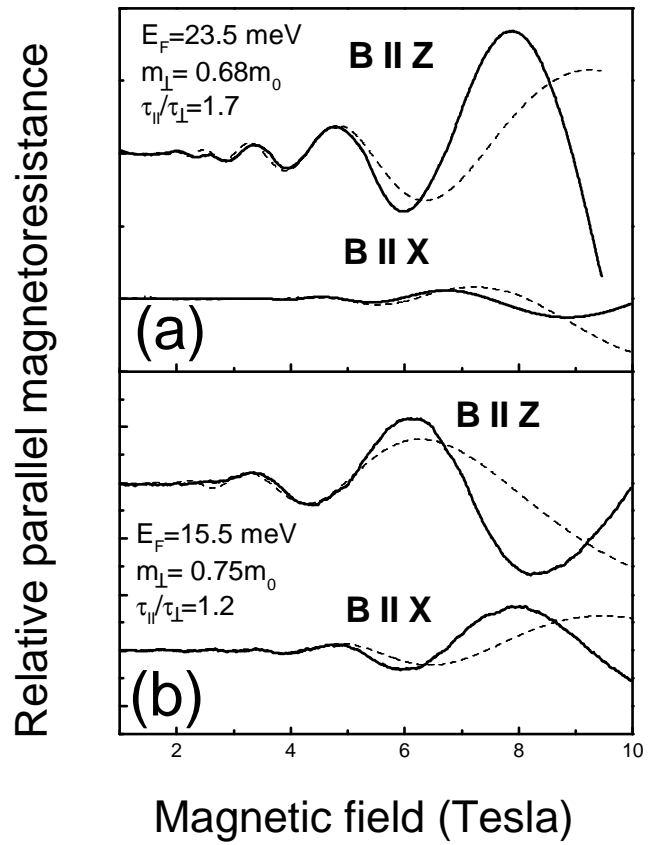

FIG. 3: Oscillatory parts of the high-field magnetoresistances measured with different orientations of the magnetic field at $T=4.2 \mathrm{~K}$ in the $(\operatorname{InGaAs})_{m}(\operatorname{InP})_{m}$ superlattices with different periods: (a) $m=6$ $M L$, (b) $m=15 M L$.

metry of the Raman lines different in the cases of the optical phonons and the coupled plasmon-like modes. Then, the shape of the Raman lines reproduces the density of states of the excitations that contribute to Raman scattering. In such a case, the coherence length of the relevant excitations may be obtained. We found the well pronounced asymmetries of the Raman lines of the $L O$ phonon of $I n P$ in the long-period SL $(m=68 M L)$ and of the $L_{3}$ coupled mode in the shortperiod SL $(m=6 \mathrm{ML})$. The fitting of the Raman intensities calculated as in Ref.[7] to the expermental spectra shown in Fig. 1 allowed us to determine the corresponding coherence lengths $\left(L_{c}\right): 0.5 \mathrm{~nm}$ and $12 \mathrm{~nm}$ for the InP longitudinal optical phonon and for the coupled $L_{3}$ mode respectively. Obviously, the obtained phonon coherence length is of the order of the size of the crystal unit cell and therefore, the phonon Raman lines did not reveal the selection rules. The much stronger coherence of the plasmon- $L O$ phonon modes resulted in their symmetrical properties determined by the crystal lattice and, as a consequence, in the corresponding selection rules.

Finally, an additional confirmation of the formation of the miniband energy structure was obtained by the high-field magnetoresistance measurements. The Shubnikov - de Haas oscillations measured with different orientations of the magnetic field (Fig. 3) demonstrate a formation of the quantized Landau levels and well pronounced anisotropy of the magnetoresistance expected in SLs. The fitting of the magnetoresistances calculated according to Ref.[8] to the experimental magnetoresistance traces allowed us to obtain the Fermi energies, the vertical effective masses $\left(m_{\perp}\right)$ and the ratios of the parallel to vertical relaxation times $\left(\tau_{\|} / \tau_{\perp}\right)$ shown in Figs.3(a,b). The calculations were performed with the parallel effective mass of $0.05 m_{0}$ corresponding to $\operatorname{In}_{0.53} G a_{0.47} A s$ [5]. The difference found between the parallel and vertical effective masses once again reveals the effect of the formation of the superlattice miniband.

Thus, clear evidences of the formation of the miniband structure in the electron energy spectrum of the InGaAs/InP SLs were observed by both the Raman scattering of the coupled plasmon - $L O$ phonon modes propagating in the growth direction of the short-period SLs and by the high-field magnetoresistance measurements.

We are grateful to H.Arakaki and C.A. de Souza for tecnical assistance. The financial support from Brazilian agencies FAPESP, CNPq and the Natural Sciences and Engineering Research Council of Canada are gratefully acknowledged.
[1] C. Hermann and C. Wesbuch, Phys.Rev.B 15, 823 (1977).

[2] A.B. Henriques, L.K. Hanamoto, R.F. Oliveira, P.L. Souza, L.C.D. Gonçalves, and B. Yavich, Braz. J. Phys. 29, 707 (1999).

[3] Light Scattering in Solids V, ed. by M. Cardona and G. Güntherodt, Springer-Verlag, Berlin, 1989.

[4] G. Bastard, Phys.Rev.B 24, 5693 (1981).

[5] I. Vurgaftman and J.R. Meyer, J.Appl.Phys. 89, 5815 (2001).
[6] Yu. Pusep, A. Milekhin, A. Toropov, Superlattices and Microstructures, 13, 115 (1993).

[7] Yu. A. Pusep, M. T. O. Silva, J. C. Galzerani, N. N. Moshegov, P. Basmaji, Phys. Rev. B 58, 10683 (1998).

[8] A.E. Stephens, D.G. Seiler, J.R. Sybert, and H.J. Mackey, Phys.Rev.B 11, 4999 (1975). 